\title{
INVARIANT SUBMODULES OF SIMPLE RINGS
}

\author{
S. A. AMITSUR
}

1. In his paper [4], I. N. Herstein states as a conjecture the following generalization of the Brauer-Cartan-Hua theorem, "If a subring $T$ of a simple ring $A$ is carried into itself by every automorphism of $A$, then $T=A$ or $T$ is contained in the center of $A$."

The results on the Lie-structure of simple rings have been applied in [2] to prove this conjecture for simple rings satisfying the descending chain condition (with one exception) (see also $[5 ; 6]$ ). The same method is taken up in this note and we prove this result for a far wider class of simple rings: simple rings which contain at least one idempotent $\neq 1$. Actually, we extend a result of Hattori [3] and Theorem 2 of [2] to these rings, from which our result follows immediately.

We show also by an example that the conjecture of Herstein is not true in the general case.

2. Let $A$ be a simple algebra over a field $F \neq$ GF [2]. In order that the results obtained here will be valid also for simple rings which do not contain a unit, we extend the meaning of "inner automorphism" to include the quasi-inner automorphism which is defined as follows: let $x \in A$ and let $x^{\prime}$ be the quasi-inverse of $x$, that is: $x+x^{\prime}+x x^{\prime}=0$; then the mapping: $y \rightarrow y+x y+y x^{\prime}+x y x^{\prime}$ is an automorphism of $A$. Such an automorphism will also be called an inner automorphism. If $A$ contains a unit, denoted by 1 , then we have $1+x^{\prime}=(1+x)^{-1}$ and $y+x y+y x^{\prime}+x y x^{\prime}=(1+x) y(1+x)^{-1}$. Thus, in this case, the preceding automorphism is inner in the usual sense.

A subspace $M$ of $A$ is said to be an invariant subspace of $A$ if $\sigma(M) \subseteq M$ for every inner automorphism $\sigma$ of $A$. We shall use the notation $[x, y]=x y-y x$, and if $C$ and $B$ are subsets of $A$ then $[C, B]$ will denote the module generated by the set $\{[c, b] \mid c \in C$, $b \in B\}$ over $F$.

Let $e \neq 1$ be an idempotent of $A$, then we set $e^{\prime}=1-e$. If $A$ does not contain the unit 1 , we shall still write $a e^{\prime}=a-a e$ and $e^{\prime} a=a-e a$ for every $a \in A$.

We shall begin with some lemmas.

Leмma 1. Let $M$ be an invariant subspace of $A$ and let $u^{2}=0, u \in A$, then $[M, u] \subseteq M$.

Received by the editors November 30, 1955. 
Indeed, the quasi-inverse of $u$ is $-u$ since $u-u-u^{2}=0$; hence, $m+u m-m u-u m u \in M$ for every $m \in M$. Since $M$ is a submodule of $A$, it follows that $u m-m u-u m u \in M$. Let $\lambda \in F \neq \mathrm{GF}$ [2] such that $\lambda \neq 0,1$. Then, since $(\lambda u)^{2}=0$ it follows that $\lambda u m-\lambda m u-\lambda^{2} u m u$ $\in M$. Consequently, $\lambda^{2}(u m-m u)-\lambda(u m-m u)=\left(\lambda^{2}-\lambda\right)[u, m] \in M$. Now $\lambda^{2}-\lambda \neq 0$; hence $[u, m] \in M$.

LEMмA 2. The elements $\{x,[x, y]\}$, where $x$ and $y$ range over the sets $e A e^{\prime}, e^{\prime}$ Ae generate the module $[A, A]$.

This lemma is essentially the difference between our proof and the proof of [2, Theorem 2].

Every element $a \in A$ can be written in the form $a=e a e+e a e^{\prime}$ $+e^{\prime} a e+e^{\prime} a e^{\prime}$. Hence the module $[A, A]$ is generated by elements of the form $[e a e, e b e],\left[e a e, e b e^{\prime}\right],\left[e a e, e^{\prime} b e\right]$ and similar elements where $e$ and $e^{\prime}$ change places. Now $\left[e a e, e b e^{\prime}\right]=e a e b e^{\prime}$; hence, since $A e A=A$ it follows that the set $e A e^{\prime}$ and similarly $e^{\prime} A e$ belongs to $[A, A]$. The elements $\left[e a e^{\prime}, e^{\prime} b e\right]$ and the similar elements are of the form required in the lemma and we just saw the elements of the form [eae, $\left.e^{\prime} b e\right]$ are in $e A e^{\prime}$ or $e^{\prime} A e$. It remains, therefore, to show that the elements $[e a e, e b e]$ and $\left[e^{\prime} a e^{\prime}, e^{\prime} b e^{\prime}\right]$ are linear combinations of elements $[x, y]$, where $x$ and $y$ are elements of $e A e^{\prime}$ and $e^{\prime} A e$. Indeed, since $A e^{\prime} A=A$, it follows that $e A e=e A e^{\prime} A e$. Hence, the elements of $e A e$ are linear combinations of elements of the form $e q e^{\prime} e^{\prime} p e$. Thus, it suffices to consider only the elements $\left[e q e^{\prime} p e, e b e\right]$. But:

$$
\left[e q e^{\prime} p e, e b e\right]=\left[e q e^{\prime}, e^{\prime} p e b e\right]-\left[e b e q e^{\prime}, e^{\prime} p e\right] \in\left[e A e^{\prime}, e^{\prime} A e\right] .
$$

A similar proof holds for elements of the form $\left[e^{\prime} a e^{\prime}, e^{\prime} b e^{\prime}\right]$.

Lemma 3. If $M$ is an invariant subspace of $A$ then $[M,[A, A]] \subseteq M$.

Indeed, let $x, y$ be two elements of the sets $e A e^{\prime}$ and $e^{\prime} A e$, then $x^{2}=y^{2}=0$. Hence, Lemma 1 shows that $[m, x]$ and $[m, y]$ belong to $M$ for every $m \in M$. Furthermore, $[m,[x, y]]=[[m, x], y]+[x$, $[m, y]]$. Hence, since $[m, x],[m, y] \in M$, it follows that $[[m, x], y]$, $[[m, y], x] \in M$ and consequently, $[m,[x, y]] \in M$. This in view of Lemma 2 yields that $[M,[A, A]] \subseteq M$.

We apply now Theorem 10a of [1], which states: if $A$ is a simple ring which is not a 4-dimensional algebra over a field of characteristic two and $T$ is a submodule of $A$ satisfying $[T,[A, A]] \subseteq T$, then either $T \supseteq[A, A]$ or $T$ is contained in the center of $A$. We apply this theorem to the module $M$, and obtain the following extension of $[2$, Theorem 2]:

Theorem 1. If $A$ is a simple algebra over a field $F \neq G F[2]$, contain- 
ing an idempotent $\neq 1$ and $A$ is not a 4-dimensional algebra over a field of characteristic two, then the invariant subspaces of $A$ either contain $[A, A]$ or they are contained in the center of $A$.

Similar to the proof of [2, Theorem 3$]$ one obtains:

Theorem 2. If $A$ satisfies the condition of Theorem 1 then the only invariant subalgebras of $A$ are $0, F$ and $A$.

The following simple proof of this theorem is due to the referee: If $S$ is an invariant subalgebra of $A$ then it follows by the previous theorem that either $S$ is contained in the center of $A$, or $S \supset[A, A]$. In the first case $S=0$ or $S=F$ and in the latter we show that $S=A$. Indeed, by Lemma 2 it follows that since $S \supset[A, A], S$ contains $e A e^{\prime}$ and $e^{\prime} A e$, hence their products $e A e^{\prime} A e=e A e$ and $e^{\prime} A e A e^{\prime}=e^{\prime} A e^{\prime}$, so that $S=A$.

3. We conclude this note with an example which disproves the conjecture of Herstein [4]:

Let $F$ be a commutative field of characteristic zero which possesses a nontrivial derivation: $g \rightarrow g^{\prime}$, e.g. $F=Q(x)$ be the field of all rational functions in a commutative indeterminate $x$ over the rational field $Q$ with the ordinary derivation $d / d x$. Consider the $\operatorname{ring} A=F[t]$ of all differential polynomials in $t$ defined by the relation $g t=t g+g^{\prime}$. This ring is well known to be a simple ring (e.g. [1]). Since the degree of the product of two polynomials of $F[t]$ is the sum of their degrees, one readily verifies that the set of all invertible elements of $F[t]$ is the field $F$. Hence $F$ is a subring of $F[t]$ which remains invariant under all automorphisms of $F[t]$. But clearly, $F D[F[t], F[t]]$ since the latter contains nonconstant polynomials, and $F$ does not belong to the center of $F[t]$ since the derivation of $F$ is nontrivial. This disproves the preceding conjecture.

\section{BIBLIOGRAPHY}

1. S. A. Amitsur, Derivations in simple rings, Proc. London Math. Soc. (To appear).

2. W. E. Baxter, Lie simplicity of a special class of associative rings, Proc. Amer. Math. Soc. vol. 7 (1956) pp. 855-863.

3. A. Hattori, On invariant subrings Jap. J. Math. vol. 21 (1951) pp. 121-129.

4. I. N. Herstein, On the Lie ring of a simple associative ring, Duke Math. J. vol. 22 (1955) pp. 471-476.

5. L. K. Hua, $A$ note on the total matrix ring over a non-commutative field, Annales de la Société Polonaise de Mathématique vol. 25 (1952) pp. 188-198.

6. F. Kasch, Invariante Untermoduln des Endomorphismenrings eine Vektoraums, Archiv der Mathematik vol. 4 (1953) pp. 182-190.

Herreiv UNiversity, Jerusalem, IsRaEl 